\title{
General Psychiatry Mental health stigma: the role of dualism, uncertainty, causation and treatability
}

\author{
Javed Latoo, ${ }^{1,2}$ Minal Mistry, ${ }^{3}$ Majid Alabdulla (D) ,, ${ }^{1,2}$ Ovais Wadoo (D) , ${ }^{1}$ Farida Jan, ${ }^{4}$ \\ Tariq Munshi, ${ }^{5}$ Yousaf Iqbal, ${ }^{1}$ Peter Haddad ${ }^{1,2}$
}

To cite: Latoo J, Mistry M, Alabdulla $\mathrm{M}$, et al. Mental health stigma: the role of dualism, uncertainty, causation and treatability. General Psychiatry 2021;34:e100498. doi:10.1136/ gpsych-2021-100498

Received 26 January 2021 Accepted 15 June 2021

Check for updates

(c) Author(s) (or their employer(s)) 2021. Re-use permitted under CC BY-NC. No commercial re-use. See rights and permissions. Published by BMJ.

${ }^{1}$ Mental Health Services, Hamad Medical Corporation, Doha, Qatar

${ }^{2}$ College of Medicine, Qatar University, Doha, Qatar ${ }^{3}$ Fredericton Addiction and Mental Health Services, Fredericton, New Brunswick, Canada

${ }^{4}$ Department of Psychiatry, Northamptonshire Healthcare NHS Foundation Trust, Northampton, UK

${ }^{5}$ Department of Psychiatry, University of Toronto, Toronto, Ontario, Canada

Correspondence to Dr Ovais Wadoo; owadoo@gmail.com

\section{INTRODUCTION}

Public stigma on mental health has been defined as 'the set of negative attitudes and beliefs that motivate individuals to fear, reject, avoid and discriminate against people with mental illness'. ${ }^{1}$ Stigma causes immense suffering to people with mental disorders across the globe. According to a recent Human Rights Watch report, people with mental health conditions are still shackled in 60 countries. $^{2}$ Nearly nine out of 10 mental health service users in England have experienced discrimination. ${ }^{3}$ Stigma can be perpetuated by people in all walks of life, including the family and friends of those with mental disorders, and healthcare professionals, including those working in mental health. The adverse consequences of stigma include shame, self-blame, isolation and discrimination. ${ }^{4}$ Examples of discrimination experienced by people with mental health disorders include others avoiding their company, and education, employment and housing opportunities being withheld. ${ }^{5}$ Stigma can deter people with mental health problems from seeking help and leads to poor adherence to treatment. People with mental disorders have high rates of physical comorbidity and higher mortality compared with the general population. ${ }^{67}$ The reasons for these health disparities are multifactorial, but discrimination and diagnostic overshadowing are believed to contribute. ${ }^{8}$ Mental health stigma has many causes including lack of knowledge and inaccurate beliefs, especially regarding the relationship between mental illness and violent behaviour. $^{9}$ In this article, we highlight mind-body dualism and an underappreciation of the similarities that can exist between mental and physical disorders as contributory factors to stigma. Negative assumptions about psychiatry include it involving uncertainty, the causation of disorders being poorly understood and conditions having poor treatability. These are misleading generalisations, but more relevant to this article is the fact that these features can be encountered in both psychiatry and general medicine.

\section{MIND-BODY DUALISM}

The brain is the most complex organ of the body, containing about 86 billion neurons and more than 100 trillion synapses. ${ }^{10}$ Its functioning is not fully understood, but advances in brain imaging and neurosciences have increased significantly in recent years and are predicted to continue. Neuroscience research shows that the mind and brain are functionally interdependent and that there is no impenetrable barrier between the two. ${ }^{11}$ In contrast, Cartesian dualism, introduced by the French philosopher René Descartes (1596-1650), placed an impenetrable wall between the brain (physical) and mind (mental), with the claim that the brain cannot create mental processes because they are two different kinds of substance (physical and mental) ${ }^{12}$ Descartes proposed that the brain is spatial and thus observable, while the mind is non-spatial and hence private. In response to critics of the theory, Descartes and his later supporters made various failed attempts to salvage dualism including a proposed role for the pineal gland and the introduction of the theories of epiphenomenalism, occasionalism and parallelism. ${ }^{13}$

Stigma has been associated with mental disorders since time immemorial, but dualism made a further contribution ${ }^{14}$ by providing a philosophical framework for an impenetrable wall between the mind and brain. In particular, it led to psychiatric and physical disorders being disorders of the mind and body, respectively, and separate in terms of aetiology and management. This may have contributed 
to the marginalisation of psychiatry from the rest of medicine as exemplified by the development of asylums in the 19th century and the split between neurology and psychiatry in the 20th century. In many countries, psychiatric units are separate from general hospitals, further perpetuating the view for patients and the public and healthcare professionals that psychiatric and physical health disorders are intrinsically different. This view is a barrier to holistic patient care, and patients with comorbid physical and psychiatric disorders are often left with inadequate treatment for one or the other category of disorder. The psychiatric/physical health divide contributes to inequities in the funding of research and clinical services, with psychiatry being the poorer relation. Mind-body dualism has led to other problems for psychiatry, including a tendency to mystify mental illness. In some cultures, psychiatric disorders are attributed to witchcraft, black magic or possession with jinn. ${ }^{15}$ Dualism contributes to the common stigmatising view that people with mental disorders can "pull themselves together', if they have a disorder of the mind, rather than the body, then it is assumed that they should be able to treat themselves. Dualism also gives an opportunity for people to offer an 'expert' opinion about the cause and management of mental illness irrespective of their professional background or training. Dualism perpetuates the clouds of uncertainty over the causality, treatability and chronicity of mental disorders, thus contributing to the growing stigma of mental health disorders compared with physical health conditions. Despite neuroscience researches confirming the interdependence of the mind and the brain, dualism remains popular among the public for various reasons, including faith, culture and poor mental health literacy.

\section{UNCERTAINTY}

Psychiatry is often viewed as involving more uncertainty in terms of aetiology, making a diagnosis, treatment and being less 'scientific' than general medicine. ${ }^{16}$ In reality, uncertainty is an inherent part of science and medicine. In science, the physical world, previously described with certainty by the principles of classical physics, is now known to be characterised by uncertainty and unpredictability at the quantum (subatomic) level as highlighted by Heisenberg's uncertainty principle. Interestingly, it has been suggested that the principles of quantum mechanics (particle-wave duality) may assist in understanding brain function. ${ }^{17}$ At the start of the 20th century, William Osler said, 'Medicine is a science of uncertainty and an art of probabilities. ${ }^{18}$ More than a 100 years later, a Lancet editorial highlighted that 'Uncertainty is inherent in healthcare, as it is in all aspects of complex behaviour in which outcomes are non-linear. Tolerance of uncertainty is a necessary skill for effective care, and self-preservation, as a doctor'. ${ }^{19}$ Uncertainty extends from diagnosis, through selecting and interpreting investigations, to making treatment decisions. However, uncertainty tends to be downplayed, and regular media reports of the latest medical breakthroughs or treatments imply more certainty and objectivity than is often the case. The evidence-based medicine movement, that started in the 1990s, has been a major advance in terms of producing systematic reviews of randomised controlled trials, developing clinical practice guidelines, promoting full disclosure of trials and campaigning against 'too much medicine' ${ }^{20}$ However, evidence-based medicine has its weaknesses. These include the limitations of clinical trials, for example, trial participants being underrepresentative in terms of age and comorbidity and most trials being short term. In addition, a restricted view of evidence-based medicine leads to a downgrading of clinical judgement and mechanistic reasoning. ${ }^{20}$ As such, evidence-based medicine has not eliminated uncertainty from the practice of medicine.

\section{CAUSATION}

The aetiology and pathophysiology of many mental disorders are poorly understood. Nevertheless, there is often a substantial evidence base for the role of risk factors, that is, biological, psychological and environmental factors that contribute to the development of mental disorders. In most cases, causation reflects the combined effect of risk factors rather than one single cause. For example, migration in childhood, urbanicity, obstetric and birth complications, and adolescent cannabis use are risk factors for schizophrenia. ${ }^{21}$ In addition, there is a well-recognised genetic contribution to schizophrenia that reflects the effect of multiple genes each carrying a small risk. ${ }^{21}$ Heterogeneity and the overlap of symptoms within different diagnostic categories contribute to difficulties in researching the causation of mental disorders. Although the causations of many physical disorders are well understood, this is not always so, and it is oversimplistic to use the level of understanding of causation to distinguish between psychiatric and medical disorders. Examples of common medical disorders whose pathophysiology/aetiology are not fully understood include ulcerative colitis, multiple sclerosis, idiopathic pulmonary fibrosis and primary hypertension. Although the cause of primary hypertension is not fully understood, there are well-recognised risk factors including overweight, high salt intake and stress, with many genetic loci also each making a small contribution to overall risk. ${ }^{22}$ The lack of a full understanding of the causes of primary hypertension does not prevent a range of effective medications being available for treatment. Similarly, although we do not know the exact causes of many psychiatric disorders, effective treatments are available including psychosocial interventions and medications.

\section{TREATABILITY}

A significant proportion of the public has a negative perception of psychiatric disorders in terms of treatability 
and longitudinal course. For example, a 2003 general population survey in the UK found that the statement 'will never recover completely' was endorsed by $25 \%$ for severe depression, $29 \%$ for alcoholism and $42 \%$ for schizophrenia. ${ }^{9}$ The same survey found that between $10 \%$ and $15 \%$ of respondents believed that people with these three disorders did not improve with treatment. In addition, the public often has a high degree of ambivalence towards psychiatric treatments. ${ }^{23}$ However, the efficacy of psychiatric treatments for a range of disorders is established by meta-analysis. This includes antidepressants for the treatment of major depression ${ }^{24}$, antipsychotics for the treatment of schizophrenia ${ }^{25}$ and cognitivebehavioural therapies for major depressive and anxiety disorders. ${ }^{26}$ Furthermore, a meta-analysis showed that the effectiveness of psychiatric drugs was broadly comparable with that of medications used to treat physical disorders. ${ }^{27}$ Chronic diseases are defined broadly as conditions that last 1 year or more and require ongoing medical attention and/or limit activities of daily living. ${ }^{28}$ Many physical illnesses are chronic, and their treatments aim at symptom alleviation rather than cure. Examples include ischaemic heart disease, diabetes mellitus, Parkinson's disease, multiple sclerosis, sarcoidosis, pulmonary fibrosis and some arthritides. In the USA, six out of 10 adults have chronic physical disorders, and these are leading causes of death and disability, and leading drivers of the nation's $\$ 3.5$ trillion annual healthcare costs. ${ }^{28}$ The reality is that both physical and mental disorders lie on spectrums regarding their courses and treatabilities. Some disorders, whether psychiatric or physical, are of short duration, whereas others are chronic/recurrent. Similarly, some physical and mental disorders respond well to treatments, others poorly and some currently have no curative treatments. Furthermore, for any specific disorder, there is individual variation in treatment responsiveness.

\section{CONCLUSIONS AND IMPLICATIONS}

As physicians, we need to change our attitude by improving our knowledge about the brain and mind. Unfortunately, people with a dualistic view of the world may strongly believe that mental disorders are untreatable, incurable and chronic in nature, as they believe that the mind cannot be explained by the functioning of the brain. Dualism supports a false division between mental and physical disorders that contributes to mental health stigma. The stigma is also partly driven by a failure to appreciate that there are more similarities than often realised between mental and physical disorders in terms of causation, management and dealing with uncertainty (box 1). Training for healthcare professionals should challenge dualism and highlight similarities that often exist between psychiatric and physical conditions. Psychiatric training for doctors should begin at medical school when attitudes are developing. ${ }^{16}$ We need to educate patients by drawing comparisons between psychiatric and physical conditions and emphasise the complex nature of psychiatric conditions, including their biopsychosocial causations,
Box 1 Common features of physical and psychiatric disorders

- Causation can be poorly understood or uncertain.

- Management often involves uncertainty.

- Course can be acute or chronic.

- Condition can often be treatable but not curable.

- Treatment may need to be short term or long term.

- Combined medications or treatments may be required.

Treatment resistance can occur.

and the pitfalls of mind-body dualism. We are not recommending a reductionist approach to mental illness. Rather, we wish to highlight that there is more common ground between mental and physical disorders that is often ignored. We think time has come to stop taking refuge in Cartesian dualism given its negative consequences highlighted in our article. It is important to avoid using ecclesiastical language to mystify mental illness, as this can create a fertile ground for uncertainty and stigma. It is essential to counter the damaging myth that psychiatric treatments are ineffective. ${ }^{9}$

Improved understanding of mental disorders can help combat the stigma associated with mental disorders among patients, carers and healthcare professionals. Greater public understanding may improve the acceptability of diagnosis and enhance adherence with treatment. Perhaps it is even worth reconsidering the distinction between psychiatry and neurology. Several authorities have argued for a merger of the two disciplines because their separation is artificial and has an adverse impact on patient management, research, funding and medical recruitment. ${ }^{29}$ It also contributes to the professional isolation of psychiatry from the rest of medicine, which further adds to the stigma of mental disorders.

Contributors All authors contributed to the conception and drafting of this commentary. JL wrote the initial draft of the manuscript; all the authors provided critical input and approved the final manuscript.

Funding The authors have not declared a specific grant for this research from any funding agency in the public, commercial or not-for-profit sectors.

Competing interests PH reports personal fees from Janssen, Lundbeck, Otsuka, NewBridge Pharmaceuticals and Sunovion, outside the submitted work.

Patient consent for publication Not required.

Provenance and peer review Not commissioned; externally peer reviewed.

Open access This is an open access article distributed in accordance with the Creative Commons Attribution Non Commercial (CC BY-NC 4.0) license, which permits others to distribute, remix, adapt, build upon this work non-commercially, and license their derivative works on different terms, provided the original work is properly cited, appropriate credit is given, any changes made indicated, and the use is non-commercial. See: http://creativecommons.org/licenses/by-nc/4.0/.

ORCID iDs

Majid Alabdulla http://orcid.org/0000-0002-3631-2650

Ovais Wadoo http://orcid.org/0000-0001-5023-9245

\section{REFERENCES}

1 Parcesepe AM, Cabassa LJ. Public stigma of mental illness in the United States: a systematic literature review. Adm Policy Ment Health 2013;40:384-99. 
2 Human Rights Watch. Living in chains: shackling of people with psychosocial disabilities worldwide, 2020. Available: https:// www.hrw.org/report/2020/10/06/living-chains/shackling-peoplepsychosocial-disabilities-worldwide [Accessed 10 Oct 2020].

3 Corker E, Hamilton S, Henderson C, et al. Experiences of discrimination among people using mental health services in England 2008-2011. Br J Psychiatry Supp/ 2013;55:s58-63.

4 Byrne P. Stigma of mental illness and ways of diminishing it. Adv Psychiatr Treat 2000;6:65-72.

5 Corrigan PW, Watson AC. Understanding the impact of stigma on people with mental illness. World Psychiatry 2002;1:16-20.

6 DE Hert M, Correll CU, Bobes J, et al. Physical illness in patients with severe mental disorders. I. Prevalence, impact of medications and disparities in health care. World Psychiatry 2011;10:52-77.

7 Walker ER, McGee RE, Druss BG. Mortality in mental disorders and global disease burden implications: a systematic review and metaanalysis. JAMA Psychiatry 2015;72:334-41.

8 Link BG, Phelan JC, Sullivan G. Mental and physical health consequences of the stigma associated with mental illnesses. The Oxford handbook of stigma, discrimination, and health, 2018.

9 Crisp A, Gelder M, Goddard E, et al. Stigmatization of people with mental illnesses: a follow-up study within the changing minds campaign of the Royal College of psychiatrists. World Psychiatry 2005;4:106-13.

10 Korade Z, Mirnics K. Programmed to be human? Neuron 2014;81:224-6.

11 Glannon W. Mind-brain dualism in psychiatry: ethical implications. Front Psychiatry 2020;11:85.

12 Thibaut F. The mind-body Cartesian dualism. Dialogues Clin Neurosci 2018;20:3.

13 Heil J. Philosophy of mind: a contemporary introduction. Routledge, 2019.

14 Ventriglio A, Bhugra D. Descartes' dogma and damage to Western psychiatry. Epidemiol Psychiatr Sci 2015;24:368-70.

15 Lim A, Hoek HW, Ghane S, et al. The attribution of mental health problems to Jinn: an explorative study in a transcultural psychiatric outpatient clinic. Front Psychiatry 2018;9:89.
16 Robertson T, Walter G, Soh N, et al. Medical students' attitudes towards a career in psychiatry before and after viewing a promotional DVD. Australas Psychiatry 2009;17:311-7.

17 Latif WA, Ggha S. Understanding neurobehavioural dynamics: a close-up view on psychiatry and quantum mechanics. Malays $J$ Med Sci 2019;26:147-56.

18 Sir William Osler: aphorisms from his bedside teachings and writings William Bennett Bean Br J Philos Sci 1954;5:172-3.

19 Lancet. Uncertainty in medicine (editorial) 2010;375:1666.

20 Sheridan DJ, Julian DG. Achievements and limitations of evidencebased medicine. J Am Coll Cardiol 2016;68:204-13.

21 Stilo SA, Murray RM. The epidemiology of schizophrenia: replacing dogma with knowledge. Dialogues Clin Neurosci 2010;12:305-15.

22 Oparil S, Acelajado MC, Bakris GL, et al. Hypertension. Nat Rev Dis Primers 2018:4:18014.

23 Dixon J, Richter D. Contemporary public perceptions of psychiatry: some problems for mental health professions. Soc Theory Health 2018;16:326-41.

24 Cipriani A, Furukawa TA, Salanti G, et al. Comparative efficacy and acceptability of 21 antidepressant drugs for the acute treatment of adults with major depressive disorder: a systematic review and network meta-analysis. Lancet 2018;391:1357-66.

25 Leucht S, Leucht C, Huhn M, et al. Sixty years of placebo-controlled antipsychotic drug trials in acute schizophrenia: systematic review, Bayesian meta-analysis, and meta-regression of efficacy predictors. AJP 2017;174:927-42.

26 Cuijpers P, Cristea IA, Karyotaki E, et al. How effective are cognitive behavior therapies for major depression and anxiety disorders? A meta-analytic update of the evidence. World Psychiatry 2016;15:245-58.

27 Leucht S, Hierl S, Kissling W, et al. Putting the efficacy of psychiatric and general medicine medication into perspective: review of metaanalyses. Br J Psychiatry 2012;200:97-106.

28 National center for chronic disease prevention and health promotion, 2020. Available: https://www.cdc.gov/chronicdisease/about/index. htm

29 Fitzgerald M. Do psychiatry and neurology need a close partnership or a merger? BJPsych Bull 2015;39:105-7.

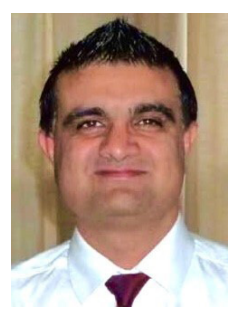

Dr Javed Latoo did his psychiatry training (residency) at University College London (UCL) and passed his membership examinations of Royal College of Psychiatrists (MRCPsych) in 2005. He received his certificate of completion of training (CCT) in 2010, from the General Medical Council (UK), after successfully completing advanced psychiatry training at the Royal Free and UCL. He worked as a consultant psychiatrist in the National Health Service (NHS) for more than 8 years before joining Hamad Medical Corporation in Qatar. Currently, he works as a senior consultant psychiatrist and a clinical director of psychiatric services in a general hospital in Doha. He is also an Assistant Professor of Psychiatry at the School of Medicine at Qatar University. He is a member of the Royal College of Psychiatrists (UK), British Neurosciences Association (UK), International Brain Research Organization (France) and Federation of European Neuroscience Societies (Germany). He is an editor of the British Journal of Medical Practitioners. Because of his interest in poetry, he also manages the UK-based peer-reviewed literary journal 'The Beautiful Space-A Journal of Art, Mind and Poetry'. His main research interests include neuropsychiatry, medical education, psychiatric aspects of COVID19, physical health in mental illness, philosophy of mind and early intervention in psychiatry. 